\title{
Note on bipartite graph tilings
}

\author{
Jan Hladký * $\quad$ Mathias Schacht ${ }^{\dagger}$
}

October 26, 2018

\begin{abstract}
Let $s<t$ be two fixed positive integers. We study sufficient minimum degree conditions for a bipartite graph $G$, with both color classes of size $n=k(s+t)$, which ensure that $G$ has a $K_{s, t^{-}}$ factor. Our result extends the work of Zhao, who determined the minimum degree threshold which guarantees that a bipartite graph has a $K_{s, s}$-factor.
\end{abstract}

\section{Introduction}

For two (finite, loopless, simple) graphs $H$ and $G$, we say that $G$ contains an $H$-factor if there exist $v(G) / v(H)$ vertex-disjoint copies of $H$ in $G$. As a synonym, we say that there exists an $H$-tiling of $G$. Obviously, if $G$ contains an $H$-factor, then $v(G)$ is a multiple of $v(H)$. For a fixed graph $H$, necessary and sufficient conditions on the minimum-degree of $G$ which guarantee that $G$ contains an $H$-factor were studied extensively. Results in this spirit include the Tutte 1-factor Theorem (see [7]), the HajnalSzemerédi Theorem [4, and series of improving results by Alon and Yuster [1, 2], Komlós [5], Zhao and Shokoufandeh 8, and by Kühn and Osthus 6]. In [6] the answer to the problem is settled (up to a constant) for any $H$. It was shown that the relevant parameters are the chromatic number and the critical chromatic number of $H$.

The additional information that $G$ is $r$-partite might help to decrease the minimum-degree threshold for $G$ containing an $H$-factor. The conjectured $r$-partite version of the Hajnal-Szemerédi Theorem 3 ] is such an example. (Recently a proof of the approximate version of the $r$-partite Hajnal-Szemerédi Theorem was announced by Csaba.) In this paper we determine the threshold for the minimum-degree of a balanced bipartite graph $G$ which guarantees that $G$ contains a $K_{s, t}$-factor, for arbitrary integers $s<t$. If the cardinalities of both color classes of $G$ are $n$, a necessary condition for $G$ having a $K_{s, t}$-factor is that $n$ is a multiple of $s+t$. The sufficient minimum-degree condition is given in Theorem 2, and a matching lower bound is provided in Theorem 3. Our work can be seen as an extension of the work of Zhao [9], who investigated the case $s=t$.

Theorem 1 (Zhao, 9]). For each $s \geq 2$ there exists a number $k_{0}$ such that if $G=(A, B ; E)$ is a bipartite graph, $|A|=|B|=n=k s$, where $k>k_{0}$, and

$$
\delta(G) \geq \begin{cases}\frac{n}{2}+s-1 & \text { if } k \text { is even }, \\ \frac{n+3 s}{2}-2 & \text { if } k \text { is odd }\end{cases}
$$

then $G$ has a $K_{s, s}$-factor.

Moreover, Zhao showed that the bounds in Theorem 1 are tight. We extend those results to $K_{s, t^{-}}$ factors with $s<t$.

\footnotetext{
*Department of Applied Mathematics, Faculty of Mathematics and Physics, Charles University, Malostranské náměstí 25, 118 00, Prague, Czech Republic and Zentrum Mathematik, Technische Universität München, Boltzmannstraße 3, D85747 Garching bei München, Germany. E-mail: honzahladky@googlemail.com. Research was supported by the Grant Agency of Charles University, and by the DFG Research Training Group 1408 "Methods for Discrete Structures" via its 2007 predoc course "Integer Points in Polyhedra".

${ }_{\dagger}^{\dagger}$ Institut für Informatik, Humboldt-Universität zu Berlin, Unter den Linden 6, D-10099 Berlin, Germany. E-mail: schacht@informatik.hu-berlin.de
} 
Theorem 2. Let $1 \leq s<t$ be fixed integers. There exists a number $k_{0} \in \mathbb{N}$ such that if $G=(A, B ; E)$ is a bipartite graph, $|A|=|B|=n=k(s+t)$, with $k>k_{0}$, and

$$
\delta(G) \geq \begin{cases}\frac{n}{2}+s-1 & \text { if } k \text { is even } \\ \frac{n+t+s}{2}-1 & \text { if } k \text { is odd }\end{cases}
$$

then $G$ has a $K_{s, t}$-factor.

On the other hand, we show that these bounds are best possible.

Theorem 3. Let $1 \leq s<t$ be fixed integers. There exists a number $k_{0} \in \mathbb{N}$ such that for every $k>k_{0}$ there exists a bipartite graph $G=(A, B ; E),|A|=|B|=k(s+t)=n$, such that

$$
\delta(G)= \begin{cases}\frac{n}{2}+s-2 & \text { if } k \text { is even, } \\ \frac{n+t+s}{2}-2 & \text { if } k \text { is odd and } t \leq 2 s+1,\end{cases}
$$

and $G$ does not have a $K_{s, t}$-factor.

The bounds in Theorem 2 and 3 exhibit a somewhat surprising phenomenon: for the case when $k$ is even the bound is independent of the value $t$, while for the case $k$ is odd, the minimum-degree condition depends on $t$. Moreover, we note that our results are not tight for the case $t>2 s+1$ and $k$ odd. We are very grateful to Andrzej Czygrinow and Louis DeBiasio for drawing our attention to an oversight in Theorem 3 in an earlier version of this note.

\section{Lower bound}

In this section we prove Theorem 3. We treat three cases (based on the parity of $k$ and on the relation between $s$ and $t$ ) separately. The proof of Theorem 3 is constructive, i.e., we will construct a graph $G$ with the demanded minimum-degree and then argue that $G$ does not contain a $K_{s, t}$-factor.

The building blocks of our constructions are the graphs $P(m, p)$, where $m, p \in \mathbb{N}$. The graphs $P(m, p)$ were introduced in [9]. We just state their properties, which will be used throughout this section.

Lemma 4. For any $p \in \mathbb{N}$ there exists a number $m_{0}$ such that for any $m \in \mathbb{N}, m>m_{0}$ there exists a bipartite graph $P(m, p)=\left(P_{1}, P_{2} ; E_{P}\right)$ satisfying

- $\left|P_{1}\right|=\left|P_{2}\right|=m$,

- $P(m, p)$ is p-regular, and

- $P(m, p)$ does not contain a copy of $K_{2,2}$.

In all constructions we assume that $n$ is large enough.

\subsection{Case $k$ is even}

For two integers $m$ and $q$ we write $Q(m, q)$ to denote (any of possibly many) bipartite graph $Q(m, q)=$ $\left(Q_{1}, Q_{2} ; E_{Q}\right)$ with the following properties:

- $\left|Q_{1}\right|=m,\left|Q_{2}\right|=m-2$,

- $Q(m, q)$ does not contain any $K_{2,2}$,

- $\operatorname{deg}(x) \in\{q-1, q\}$ for any vertex $x \in Q_{1}$, and

- $\operatorname{deg}(y)=q$ for any vertex $y \in Q_{2}$.

Such graphs $Q(m, q)$ do exist for fixed $q$ and large $m$. One way to construct them is by taking the graph $P(m, q)=\left(P_{1}, P_{2} ; E_{P}\right)$ from Lemma 4 selecting two vertices $w_{1}, w_{2} \in P_{2}$ such that they do not share a common neighbor in $P_{1}$, and then take $Q(m, q)$ to be the subgraph of $P(m, q)$ induced by the vertex sets $P_{1}, P_{2} \backslash\left\{w_{1}, w_{2}\right\}$. In particular, the graph $Q(m, 0)$ is the empty graph.

Now we describe the construction of the graph $G$. Partition $A=A_{1}+A_{2}, B=B_{1}+B_{2},\left|A_{1}\right|=$ $\left|B_{1}\right|=\frac{n}{2}+1,\left|A_{2}\right|=\left|B_{2}\right|=\frac{n}{2}-1$. The graph $G$ is described by 
- $G\left[A_{i}, B_{i}\right]$ is a complete bipartite graph for $i=1,2$, and

- $G\left[A_{1}, B_{2}\right] \cong G\left[B_{1}, A_{2}\right] \cong Q(n / 2+1, s-1)$.

We have $\delta(G)=\frac{n}{2}+s-2$. The fact that there exists no $K_{s, t}$-factor is implied immediately by the fact that there is no subgraph isomorphic to $K_{s, t}$ whose vertices would touch both $A_{1}$ and $B_{2}$, or $A_{2}$ and $B_{1}$.

2.2 Case $k$ is odd, $2 s+1 \geq t>s+1$

Let $k=2 l+1, n=k(s+t)$. Note that $\frac{n-t+s+2}{2}$ is an integer. Partition $A=A_{1}+A_{2}+A_{*}, B=$ $B_{1}+B_{2}+B_{*},\left|A_{1}\right|=\left|A_{2}\right|=\left|B_{1}\right|=\left|B_{2}\right|=\frac{n-t^{2}+s+2}{2},\left|A_{*}\right|=\left|B_{*}\right|=t-s-2$. The graph $G$ is described by

- $G\left[A_{i}, B_{i}\right]$ is a complete bipartite graph for $i=1,2$,

- $G\left[A_{*}, B_{i}\right]$ and $G\left[B_{*}, A_{i}\right]$ are complete bipartite graphs for $i=1,2$,

- $G\left[A_{1}, B_{2}\right] \cong G\left[A_{2}, B_{1}\right] \cong P\left(\frac{n-t+s+2}{2}, s-1\right)$,

- the graph $G\left[A_{*}, B_{*}\right]$ is empty.

We have $\delta(G)=\frac{n+t+s}{2}-2$. To see that $G$ does not have a $K_{s, t}$-factor, we argue as follows. Suppose for contradiction that $G$ has a $K_{s, t}$-factor. Fix a $K_{s, t}$-factor of $G$. First, observe that there cannot be a copy isomorphic to $K_{s, t}$ intersecting both $A_{1} \cup B_{1}$ and $A_{2} \cup B_{2}$. Let $r_{1}$ and $r_{2}$ be the number of copies of $K_{s, t}$ in the tiling whose color class of size $t$ touches $A_{1}$ and $B_{1}$, respectively. Let $A_{c}$ and $B_{c}$ be vertices covered by these $r_{1}+r_{2}$ copies. It holds

$$
A_{1} \subset A_{c} \subset A_{1} \cup A_{*} \text { and } \quad B_{1} \subset B_{c} \subset B_{1} \cup B_{*} .
$$

If $r_{1} \neq r_{2}$ then ||$A_{c}|-| B_{c}|| \geq t-s$, which contradicts (1). Thus, $r_{1}=r_{2}$. We conclude that

$$
\frac{l(s+t)+s+1}{s+t} \leq r_{1} \leq \frac{l(s+t)+t-1}{s+t}
$$

a contradiction to the integrality of $r_{1}$.

\subsection{Case $k$ is odd, $t=s+1$}

By $R(m, q)$ we denote (any of possibly many) bipartite graph $R(m, q)=\left(R_{1}, R_{2} ; E_{R}\right)$ with the following properties:

- $\left|R_{1}\right|=m,\left|R_{2}\right|=m-1$,

- $R(m, q)$ does not contain any $K_{2,2}$,

- for any vertex $x$ in $R_{1}$, it holds $\operatorname{deg}(x) \in\{q-1, q\}$, and

- for any vertex $y$ in $R_{2}$, it holds $\operatorname{deg}(y)=q$.

For fixed $q$ and large $m$ the existence of such a graph $R(m, q)$ follows by a construction analogous to the construction of the graph $Q(m, q)$.

Let $k=2 l+1$. Partition $A=A_{1}+A_{2}, B=B_{1}+B_{2},\left|A_{1}\right|=\left|B_{1}\right|=l(s+t)+s,\left|A_{2}\right|=\left|B_{2}\right|=$ $l(s+t)+s+1$. The graph $G$ is described by

- $G\left[A_{i}, B_{i}\right]$ is a complete bipartite graph for $i=1,2$,

- $G\left[B_{2}, A_{1}\right] \cong G\left[A_{2}, B_{1}\right] \cong R((n+1) / 2, s-1)$.

One immediately sees that $\delta(G)=\frac{n+t+s}{2}-2$ and no $K_{s, t}$-tiling of $G$ exists. 


\section{$3 \quad$ Upper bound}

We prove Theorem 2 in this section. The proof of Theorem 2 utilizes the previous work of Zhao [9. We will need the following lemma, which allows us to find many vertex disjoint copies of certain stars. For $h \in \mathbb{N}$, an $h$-star is a graph $K_{1, h}$, its center is the unique vertex in the part of size one. Moreover, for a graph $G$ and two disjoint sets $A, B \subset V(G)$ we define

$$
\delta(A, B)=\min \{\operatorname{deg}(v, B): v \in A\}, \quad \Delta(A, B)=\max \{\operatorname{deg}(v, B): v \in A\}
$$

and

$$
d(A, B)=\frac{e(A, B)}{|A||B|} .
$$

Lemma 5 (Zhao, [9]). Let $1 \leq h \leq \delta \leq M$ and $0<c<1 /(6 h+7)$. Suppose that $H=\left(U_{1}, U_{2} ; E_{H}\right)$ is a bipartite graph such that ||$U_{i}|-M| \leq c M$ for $i=1,2$. If $\delta=\delta\left(U_{1}, U_{2}\right) \leq c M$ and $\Delta=\Delta\left(V_{2}, V_{1}\right) \leq c M$, then we can find a family of vertex-disjoint $h$-stars, $2(\delta-h+1)$ of which have centers in $U_{1}$ and $2(\delta-h+1)$ of which have centers in $U_{2}$.

As in [9] we distinguish between an extremal and a non-extremal case. If we find a $K_{s+t, s+t}$-factor in $G$ we are done, as each copy of $K_{s+t, s+t}$ can be split into two copies of $K_{s, t}$ and hence we have a $K_{s, t}$-factor. Thus the theorem stated next is just a corollary of [9, Theorem 4.1].

Theorem 6 (Zhao, 9]). For every $\alpha>0$ and positive integers $s<t$, there exist $\beta>0$ and a positive integer $k_{0}$ such that the following holds for all $n=k(s+t)$ with $k>k_{0}$. Given a bipartite graph $G=(A, B ; E)$ with $|A|=|B|=n$, if $\delta(G)>\left(\frac{1}{2}-\beta\right) n$, then either $G$ contains a $K_{s, t}$-factor, or there exist

$$
A_{1} \subset A, \quad B_{1} \subset B \quad \text { such that }\left|A_{1}\right|=\left|B_{1}\right|=\lfloor n / 2\rfloor, \quad d\left(A_{1}, B_{1}\right)<\alpha .
$$

Therefore, we reduce the problem to the extremal case. Let $\alpha=\alpha(t)>0$ be small. As in the proof of Theorem 11 in 9], define

$$
\begin{array}{ll}
A_{1}^{\prime}=\left\{x \in A: \operatorname{deg}\left(x, B_{1}\right)<\alpha^{\frac{1}{3}} \frac{n}{2}\right\}, & B_{1}^{\prime}=\left\{x \in B: \operatorname{deg}\left(x, A_{1}\right)<\alpha^{\frac{1}{3}} \frac{n}{2}\right\}, \\
A_{2}^{\prime}=\left\{x \in A: \operatorname{deg}\left(x, B_{1}\right)>\left(1-\alpha^{\frac{1}{3}}\right) \frac{n}{2}\right\}, & B_{2}^{\prime}=\left\{x \in B: \operatorname{deg}\left(x, A_{1}\right)>\left(1-\alpha^{\frac{1}{3}}\right) \frac{n}{2}\right\}, \\
A_{0}=A-A_{1}^{\prime}-A_{2}^{\prime}, & B_{0}=B-B_{1}^{\prime}-B_{2}^{\prime}, \\
G_{1}=G\left[A_{1}^{\prime}, B_{1}^{\prime}\right], & G_{2}=G\left[A_{2}^{\prime}, B_{2}^{\prime}\right] .
\end{array}
$$

Similarly as in the proof of Theorem 11 in 9 , we assume that removing any edge from $G$ would violate the minimum-degree condition and then change $A_{i}^{\prime}$ and $B_{i}^{\prime}$ a little so that $\Delta\left(G_{1}\right), \Delta\left(G_{2}\right)<\alpha^{\frac{1}{9}} n$. Vertices in $A_{0} \cup B_{0}$ are called special.

\section{1 $k$ is even}

To exhibit the existence of a tiling in this case, it is sufficient to translate carefully the proof of Case I of Theorem 11 from [9]. We give a sketch of the proof below and refer the reader to the corresponding places in 9 for more details.

Set $\mathcal{V}=\left(A_{1}^{\prime}, B_{1}^{\prime}, A_{2}^{\prime}, B_{2}^{\prime}\right)$. First assume, that no member of $\mathcal{V}$ contains more than $n / 2$ vertices. We add vertices from $A_{0}$ and $B_{0}$ into sets of $\mathcal{V}$ in such a way, that every set has size exactly $n / 2$. Then, we may apply arguments used in [9], based on Hall's Marriage Theorem, to find a $K_{s+t, s+t}$ tiling.

Next, assume that there is only one set in $\mathcal{V}$ which has more than $n / 2$ elements. Without loss of generality, assume that it is $A_{2}^{\prime}$, i.e., $\left|A_{2}^{\prime}\right|=c>n / 2$. Lemma 5 applied to the graph $G\left[A_{2}^{\prime}, B_{2}^{\prime}\right]$ yields the existence of $c-n / 2$ disjoint $s$-stars with centers in $A_{2}^{\prime}$. We move the centers of the stars into $A_{1}^{\prime}$ and extend each of the stars into a copy of $K_{s, t}$ (each of these copies lies entirely in $A_{1}^{\prime} \cup B_{2}^{\prime}$, with the color class of size $s$ being contained in $B_{2}^{\prime}$ ). We distribute vertices of $B_{0}$ into $B_{1}^{\prime}$ and $B_{2}^{\prime}$ so, that $\left|B_{1}^{\prime}\right|=\left|B_{2}^{\prime}\right|=n / 2$. Then, it is easy to finish the entire tiling. This is done in three steps. In the first step, we find in an arbitrary manner $c-n / 2$ copies of $K_{s, t}$ (disjoint with the previous ones) in $G\left[A_{1}^{\prime}, B_{2}^{\prime}\right]$ placed in such a way, that the color-class of size $s$ lies in $A_{1}^{\prime}$. This step ensures us, that the cardinalities of untiled (i.e., those vertices which are not covered by the partial $K_{s, t}$-factor) vertices in the both color-classes of $G\left[A_{1}^{\prime}, B_{2}^{\prime}\right]$ are equal and divisible by $s+t$. In the second step, all yet untiled 
vertices of $G\left[A_{1}^{\prime}, B_{2}^{\prime}\right]$ which were originally special vertices are tiled. In the third step, the tiling is in an analogous manner defined for $G\left[A_{2}^{\prime}, B_{1}^{\prime}\right]$.

Now, assume that two diagonal sets of $\mathcal{V}$, say $A_{2}^{\prime}$ and $B_{1}^{\prime}$ have sizes more than $n / 2$. Then we apply separately Lemma 5 to $G\left[A_{2}^{\prime}, B_{2}^{\prime}\right]$ and $G\left[A_{1}^{\prime}, B_{1}^{\prime}\right]$ to obtain families $\mathcal{S}_{A}$ and $\mathcal{S}_{B}$ of disjoint $s$-stars with centers in $A_{2}^{\prime}$ and $B_{1}^{\prime}$, such that $\left|A_{2}^{\prime}\right|-\left|\mathcal{S}_{A}\right|=\left|B_{1}^{\prime}\right|-\left|\mathcal{S}_{B}\right|=n / 2$. We move the centers of the stars to $A_{1}^{\prime}$ and $B_{2}^{\prime}$ and proceed as in the previous case.

The remaining case is when two non-diagonal sets from $\mathcal{V}$ have size more than $n / 2$. Assume these are $A_{2}^{\prime}$ and $B_{1}^{\prime}$. We apply Lemma 5 to the graph $G\left[A_{2}^{\prime}, B_{2}^{\prime}\right]$ to obtain families $\mathcal{S}_{A}$, $\mathcal{S}_{B}$ of disjoint $s$-stars with centers in $A_{2}^{\prime}$ and $B_{2}^{\prime}$, such that $\left|A_{2}^{\prime}\right|-\left|\mathcal{S}_{A}\right|=\left|B_{2}^{\prime}\right|-\left|\mathcal{S}_{B}\right|=n / 2$. We proceed as in the previous cases.

\section{$3.2 k$ is odd}

Let $k=2 l+1$. We say that a set of special vertices $\left(A_{0}\right.$ and/or $\left.B_{0}\right)$ is small if its size is less than $t-s$. Otherwise, it is called big.

We distinguish four cases.

- Both $A_{0}$ and $B_{0}$ are small. Then there exist $i, j \in\{1,2\}$, such that $\left|A_{i}^{\prime}\right|,\left|B_{j}^{\prime}\right| \geq l(s+t)+s+1$. If $i=j$, then we apply Lemma 5 to the graph $G_{i}$ and find families $\mathcal{S}_{A}, \mathcal{S}_{B}$ of pairwise disjoint $s$-stars with centers in $A_{i}^{\prime}$ and $B_{i}^{\prime}$ respectively, so that $\left|A_{i}^{\prime}\right|-\left|\mathcal{S}_{A}\right|=\left|B_{i}^{\prime}\right|-\left|\mathcal{S}_{B}\right|=l(s+t)+s$. Move the centers of the stars in $A_{3-i}^{\prime}$ and $B_{3-i}^{\prime}$. After the changes we shall tile two graphs: $G\left[A_{1}^{\prime}, B_{2}^{\prime}\right]$ and $G\left[A_{2}^{\prime}, B_{1}^{\prime}\right]$. Note, that both those graphs are not balanced. The tiling procedure is analogous to the previous cases (when $k$ is even); the only difference is that one copy of $K_{s, t}$ has to be found in the graphs first to make each of them balanced.

If $i \neq j$, we can assume that $\left|A_{j}^{\prime}\right|,\left|B_{i}^{\prime}\right| \leq l(s+t)+s$. Since if this does not hold, then we could change one index and continue as in the case when $i=j$. We will show that one can add vertices to $A_{j}^{\prime}$ and to $B_{i}^{\prime}$ so that $\left|A_{j}^{\prime}\right|=l(s+t)+s$ and $\left|B_{i}^{\prime}\right|=l(s+t)+t$. Then, the existence of the tiling will follow by standard arguments. We apply Lemma 5 to the graph $G_{j}$ to obtain a family of $\left|B_{j}^{\prime}\right|-(l(s+t)+s)$ vertex disjoint $s$-stars with centers in $B_{j}^{\prime}$ and end-vertices in $A_{j}^{\prime}$. If we moved all the centers to $B_{i}^{\prime}$ and all the vertices of $B_{0}$, the cardinality of $B_{i}^{\prime}$ would be

$$
\left|B_{i}^{\prime}\right|+\left(\left|B_{j}^{\prime}\right|-(l(s+t)+s)\right)+\left|B_{0}\right|=l(s+t)+t .
$$

The same applies for $A_{j}^{\prime}$. Therefore, by removing some of the vertices, we may attain $\left|A_{j}^{\prime}\right|=$ $l(s+t)+s$ and $\left|B_{i}^{\prime}\right|=l(s+t)+t$. Then, the existence of a tiling follows.

- $A_{0}$ is small and $B_{0}$ is big. Then at least one $B_{i}^{\prime}$ (say $B_{2}^{\prime}$ ) has at most $l(s+t)+s$ vertices. Lemma 5 asserts that we can find a family $\mathcal{S}_{B}$ of disjoint $s$-stars with centers in $B_{1}^{\prime}$ and end-vertices in $A_{1}^{\prime}$, such that $\left|B_{1}^{\prime}\right|-\left|\mathcal{S}_{B}\right| \leq l(s+t)+s$. This implies, that we can find vertices (in $B_{0}$ or centers of the stars of $\left.\mathcal{S}_{B}\right)$ which can be moved to $B_{2}^{\prime}$ so that $\left|B_{2}^{\prime}\right|=l(s+t)+t$.

As $A_{0}$ is small, one of $A_{1}^{\prime}$ and $A_{2}^{\prime}$ must have at least $l(s+t)+s+1$ vertices. The tiling can be found by standard arguments if we achieve to have $\left|A_{1}^{\prime}\right|=l(s+t)+s$. If $\left|A_{1}^{\prime}\right|>l(s+t)+s$, Lemma 5 yields existence of a family $\mathcal{S}_{A}$ of disjoint $s$-stars with centers in $A_{1}^{\prime}$ and end-vertices in $B_{1}^{\prime}$ such that $\left|A_{1}^{\prime}\right|-\left|\mathcal{S}_{A}\right|=l(s+t)+s$. Moving the centers to $A_{2}^{\prime}$, we achieve $\left|A_{1}^{\prime}\right|=l(s+t)+s$. Assume that $\left|A_{1}^{\prime}\right| \leq l(s+t)+s$. The size of $A_{2}^{\prime}$ is $k(s+t)-\left|A_{1}^{\prime}\right|-\left|A_{0}\right|>l(s+t)+s$. Lemma 5yields existence of a family $\mathcal{S}_{A}$ of disjoint $s$-stars in $G_{2}$ centered in $A_{2}^{\prime}$ with the property that $\left|A_{1}^{\prime}\right|+\left|\mathcal{S}_{A}\right|=l(s+t)+s$. Moving the centers to $A_{1}^{\prime}$ yields demanded $A_{1}^{\prime}=l(s+t)+s$.

- $A_{0}$ is big and $B_{0}$ is small. The analysis of this case is analogous to the previous one.

- Both $A_{0}$ and $B_{0}$ are big. We shall show in the next paragraph, that we can achieve $A_{1}^{\prime}$ to be of size $l(s+t)+s$ and of size $l(s+t)+t$. An analogous procedure can be used to show the same property for the set $B_{1}^{\prime}$. Then, the existence of the tiling follows immediately; one prescribes the cardinalities of $A_{1}^{\prime}$ and $B_{1}^{\prime}$ to be equal to the same number $l(s+t)+s$.

If $\left|A_{i}^{\prime} \cup A_{0}\right|<l(s+t)+t$ for some $i \in\{1,2\}$, then we have $\left|A_{3-i}^{\prime}\right|>l(s+t)+s$. Appealing to Lemma 5 we can remove centers of $s$-stars from $A_{3-i}^{\prime}$ in such a way that $\left|A_{3-i}^{\prime}\right|=l(s+t)+s$. 
Also, by moving $t-s$ vertices from the big set $A_{0}$ to $A_{3-i}^{\prime}$ arrive at $\left|A_{3-i}^{\prime}\right|=l(s+t)+t$. Then, the partial $K_{s, t}$-tiling can be extended to a $K_{s, t}$-factor.

Finally, if both $\left|A_{1}^{\prime}\right| \leq l(s+t)+s$ and $\left|A_{2}^{\prime}\right| \leq l(s+t)+s$ then we redistribute some vertices (again, appealing to Lemma 5, and using the set $\left.A_{0}\right)$ to arrive at the situation when $\left|A_{1}^{\prime}\right|=l(s+t)+s$, $\left|A_{2}^{\prime}\right|=l(s+t)+t$. Then the tiling can be extended as before.

\section{Acknowledgement}

We thank a careful referee for suggesting several improvements in the presentation.

\section{References}

[1] N. Alon and R. Yuster. Almost H-factors in dense graphs. Graphs Combin., 8(2):95-102, 1992.

[2] N. Alon and R. Yuster. H-factors in dense graphs. J. Combin. Theory Ser. B, 66(2):269-282, 1996.

[3] E. Fischer. Variants of the Hajnal-Szemerédi theorem. J. Graph Theory, 31(4):275-282, 1999.

[4] A. Hajnal and E. Szemerédi. Proof of a conjecture of P. Erdős. In Combinatorial theory and its applications, II (Proc. Colloq., Balatonfüred, 1969), pages 601-623. North-Holland, Amsterdam, 1970.

[5] J. Komlós. Tiling Turán theorems. Combinatorica, 20(2):203-218, 2000.

[6] D. Kühn and D. Osthus. The minimum degree threshold for perfect graph packings. Combinatorica, 29(1):65-107, 2009.

[7] L. Lovász and M. D. Plummer. Matching theory, volume 121 of North-Holland Mathematics Studies. North-Holland Publishing Co., Amsterdam, 1986. Annals of Discrete Mathematics, 29.

[8] A. Shokoufandeh and Y. Zhao. Proof of a tiling conjecture of Komlós. Random Structures Algorithms, 23(2):180-205, 2003.

[9] Y. Zhao. Bipartite graph tiling. SIAM J. Discrete Math., 23(2):888-900, 2009. 